\title{
A global survey of potential acceptance of a COVID-19 vaccine
}

\author{
Jeffrey V. Lazarus ${ }^{1 凶}$, Scott C. Ratzan², Adam Palayew', Lawrence O. Gostin ${ }^{3}$, Heidi J. Larson ${ }^{4}$, \\ Kenneth Rabin ${ }^{2}$, Spencer Kimball ${ }^{5}$ and Ayman El-Mohandes ${ }^{2}$
}

\begin{abstract}
Several coronavirus disease 2019 (COVID-19) vaccines are currently in human trials. In June 2020 , we surveyed 13,426 people in 19 countries to determine potential acceptance rates and factors influencing acceptance of a COVID-19 vaccine. Of these, $71.5 \%$ of participants reported that they would be very or somewhat likely to take a COVID-19 vaccine, and 48.1\% reported that they would accept their employer's recommendation to do so. Differences in acceptance rates ranged from almost $\mathbf{9 0 \%}$ (in China) to less than $\mathbf{5 5 \%}$ (in Russia). Respondents reporting higher levels of trust in information from government sources were more likely to accept a vaccine and take their employer's advice to do so.

The COVID-19 pandemic is expected to continue to impose enormous burdens of morbidity and mortality while severely disrupting societies and economies worldwide. Governments must be ready to ensure large-scale, equitable access and distribution of a COVID-19 vaccine if and when a safe and effective one becomes available. This will require sufficient health system capacity, as well as strategies to enhance trust in and acceptance of the vaccine and those who deliver it.
\end{abstract}

In 2015, the World Health Organization (WHO) Strategic Advisory Group of Experts on Immunization defined vaccine hesitancy as a 'delay in acceptance or refusal of vaccination despite availability of vaccination services ${ }^{\prime}$, which can vary in form and intensity based on when and where it occurs and what vaccine is involved, as has been confirmed in multiple studies ${ }^{2,3}$. Concern about vaccine hesitancy is growing worldwide ${ }^{4}$; in fact, WHO identified it as one of the top ten global health threats in 2019 (https://www.who. int/news-room/spotlight/ ten-threats-to-global-health-in-2019). In many countries, vaccine hesitancy and misinformation present substantial obstacles to achieving coverage and community immunity ${ }^{5,6}$.

Governments, public health officials and advocacy groups must be prepared to address hesitancy and build vaccine literacy so that the public will accept immunization when appropriate. Anti-vaccination activists are already campaigning in multiple countries against the need for a vaccine, with some denying the existence of COVID-19 altogether ${ }^{7}$. Misinformation spread through multiple channels could have a considerable effect on the acceptance of a COVID-19 vaccine ${ }^{8}$. The accelerated pace of vaccine development has further heightened public anxieties and could compromise acceptance.

Governments and societies must gauge current levels of willingness to receive a potentially safe and effective COVID19 vaccine and identify correlates of vaccine hesitancy and/or acceptance. We present findings from a survey of the likelihood of vaccine acceptance from a sample of 13,426 respondents in 19 countries.

\section{Results}

Survey respondents represented a random sample of the populations of 19 countries that comprise around $55 \%$ of the global population. Their characteristics and a summary of their responses to the survey questions are listed in Table 1 . Women were $53.5 \%$ of the study population, and $63.3 \%$ of all participants earned more than $\$ 32$ per day. More than a third of the respondents (36.3\%) had a university degree, and $62.4 \%$ were between 25 and 54 years old.

Respondents from China gave the highest proportion of positive responses (631 of 712 respondants, $88.6 \%$ ) and the lowest proportion of negative responses ( 5 of $712,0.7 \%$ ) when asked if they would take a 'proven, safe and effective vaccine'. Respondents from Poland reported the highest proportion of negative responses (182 of 666, $27.3 \%$ ), whereas Russian respondents gave the lowest proportion of positive responses (373 of $680,54.9 \%$ ). The proportion of positive responses for all countries can be found in Extended Data Fig. 1.

When asked whether "You would accept a vaccine if it were recommended by your employer and was approved safe and effective by the government," $14.0 \%(1,881$ of 13,426$)$ completely agreed, whereas $25.9 \%(3,478$ of 13,426$)$ somewhat or completely disagreed (Table 1). There was considerable variation by country, with China again having the highest proportion of positive responses (596 of $712,83.7 \%$ ) and the lowest proportion of negative responses (26 of $712,3.7 \%)$. Russia had the highest proportion of negative responses (278 of $680,40.9 \%)$ and the lowest proportion of respondents (184 of $680,27.1 \%$ ) willing to accept their employer's recommendation (Supplementary Table 1).

Table 2 summarizes results for the 16 regressions: one set of eight univariate regressions for the positive outcomes for the two questions of interest against the four demographic variables: whether or not the respondent or a family member was sick, cases per million categorized as high/medium/low, mortality per million categorized as high/medium/low and trust in government. People aged $25-54,55-64$ and $65+$ were more likely to accept the vaccine than those who were aged 18-24. This difference was strongest (odds ratio $(\mathrm{OR})=1.73 ; 95 \%$ confidence interval $(\mathrm{CI})(1.48,2.02)$ ) when responses from the oldest age cohort and those from the youngest age cohort were compared (Table 2). A similar trend was observed in regard to acceptance of the vaccine if one's employer required it, except that responses from the oldest age cohort were similar to the youngest age cohort. Gender differences were small, but the univariate association for both questions suggested that men were

'Barcelona Institute for Global Health (ISGlobal), Hospital Clínic, University of Barcelona, Barcelona, Spain. ${ }^{2}$ City University of New York (CUNY) Graduate School of Public Health \& Health Policy, New York NY, USA. ${ }^{3}$ Georgetown University, Washington, DC, USA. ${ }^{4}$ London School of Hygiene and Tropical Medicine, London, United Kingdom. ${ }^{5}$ Emerson College, Boston, MA, USA. ${ }_{e}$-mail: jeffrey.lazarus@isglobal.org 
Table 1 | Description of participants and breakdown of the two COVID-19 vaccine questions

\begin{tabular}{ll} 
& Overall \\
\hline$n$ & 13,426 \\
\hline
\end{tabular}

Gender (\%)

Female

$7,172(53.5)$

Male

Other

Gapminder income level (\%)

$<$ US\$ 2 per day

$447(3.3)$

$\$ 2-\$ 8$ per day

$840(6.3)$

$\$ 8-\$ 32$ per day

$\$ 32+$ per day

$8,498(63.3)$

Did not answer

$630(4.7)$

Education level (\%)

Less than high school

$3,830(28.6)$

High school or some college

$4,692(35.0)$

Bachelor's degree

$3,694(27.6)$

Postgraduate degree

$1,179(8.8)$

Age group in years (\%)

$\begin{array}{ll}18-24 & 2,057(15.4) \\ 25-54 & 8,360(62.4) \\ 55-64 & 1,493(11.1) \\ 65+ & 1,485(11.1)\end{array}$

Accept COVID-19 vaccine if generally available (\%)

Completely agree

$6,288(46.8)$

Somewhat agree

$3,316(24.7)$

Neutral/no opinion

$1,912(14.2)$

Somewhat disagree

819 (6.1)

Completely disagree

1,091 (8.1)

Accept COVID-19 vaccine if employer recommended it (\%)

Completely agree

$1,881(14.0)$

Somewhat agree

$4,579(34.1)$

Neutral/no opinion

$3,488(26.0)$

Somewhat disagree

$2,299(17.1)$

Completely disagree

$1,179(8.8)$

slightly less likely to respond positively than women, with an OR of $0.84(95 \%$ CI $(0.78,0.91))$ of men responding positively relative to women for the general question, and an OR of 0.93 (95\% CI $(0.87$, 1.00)) for the business-related question.

People earning more than $\$ 32$ per day were 2.18 (95 CI\% (1.79, 2.64)) times more likely to respond positively to the general question than people earning less than \$2 per day. Higher levels of education were also associated positively with vaccine acceptance on both questions. People who reported COVID-19 sickness in themselves or family members were no more likely to respond positively to the vaccine question than other respondents $(\mathrm{OR}=0.97 ; 95 \% \mathrm{CI}$ $(0.87,1.08))$.

Respondents who said that they trusted their government were more likely to accept a vaccine than those who said that they did not $(\mathrm{OR}=1.67 ; 95 \% \mathrm{CI}(1.54,1.80))$. Moreover, if an individual trusted their government, they were more likely to respond positively to their employer's vaccine recommendation than someone who did $\operatorname{not}(\mathrm{OR}=4.35 ; 95 \% \mathrm{CI}(4.01,4.72))$ (Table 2$)$.

\section{Discussion}

We conducted a study of potential acceptance of a COVID-19 vaccine in 13,426 randomly selected individuals across 19 countries, most with a high COVID-19 burden. Of these, $71.5 \%$ responded that they would take a vaccine if it were proven safe and effective, and $48.1 \%$ said that they would get vaccinated if their employer recommended it (Table 1). However, we observed high heterogeneity in responses between countries. Furthermore, reporting one's willingness to get vaccinated might not be necessarily a good predictor of acceptance, as vaccine decisions are multifactorial and can change over time.

The far-from-universal willingness to accept a COVID-19 vaccine is a cause for concern. Countries where acceptance exceeded $80 \%$ tended to be Asian nations with strong trust in central governments (China, South Korea and Singapore). A relatively high tendency toward acceptance in middle-income countries, such as Brazil, India and South Africa, was also observed. Unless and until the origins of such wide variation in willingness to accept a COVID-19 vaccine is better understood and addressed, differences in vaccine coverage between countries could potentially delay global control of the pandemic and the ensuing societal and economic recovery.

Univariate variation among demographically defined groups was least among those with lower education and income levels. Future vaccine communication strategies should consider the level of health, scientific and general literacy in subpopulations, identify locally trusted sources of information ${ }^{10}$ and go beyond simply pronouncing that vaccines are safe and effective. Strategies to build vaccine literacy and acceptance should directly address community-specific concerns or misconceptions, address historic issues breeding distrust and be sensitive to religious or philosophical beliefs ${ }^{11}$. Researchers have identified promising interventions for building confidence and reducing vaccine hesitancy in different contexts $^{12,13}$, but translating this evidence into large-scale vaccination campaigns will require particular awareness of and attention to existing public perceptions and felt needs. Engaging formal and informal opinion leaders within these communities will be key.

Additionally, we observed age-related associations with vaccine acceptance. Older people were more likely to report that they would take a vaccine, whereas respondents 25-54 and 55-64 years of age were more likely to accept an employer's vaccine recommendation. This finding might reflect who was actually employed or employable at the time of the survey-an issue we did not investigate. Men in this study were less likely than women to accept vaccines in general or their employer's recommendation to get vaccinated; however, this association was not strong. Those with a higher income were most likely to accept a vaccine than those with a lower income. These data could help governments, policymakers, health professionals and international organizations to more effectively target messaging around COVID-19 vaccination programs.

The other source of concern was a discrepancy between reported acceptance of a COVID-19 vaccine and acceptance if vaccination was mandated by one's employer. All respondents, regardless of nationality, reported that they would be less likely to accept a COVID-19 vaccine if it were mandated by employers. This finding across all countries with both high and low reported vaccine acceptance proportions suggests that promoting voluntary acceptance is a better option for employers. It might seem easier to monitor compliance among adults in the working age group if employers required it, but this could fail if it is perceived as limiting employees' freedom of choice or a manifestation of employers' self-interest ${ }^{14}$.

A careful balance is required between educating the public about the necessity for universal vaccine coverage and avoiding any suggestion of coercion. Respected community-based groups and non-governmental organizations, such as the Red Cross, which is considered to be impartial, are essential to help build trust in a future COVID-19 vaccine. 
Table 2 | Univariate regression outputs for vaccine acceptability questions against demographics and variables of interest

\begin{tabular}{|c|c|c|}
\hline & Beta-coefficients of vaccine questions ( $95 \% \mathrm{Cls}$ ) & Beta-coefficients of business question ( $95 \% \mathrm{Cls}$ ) \\
\hline \multirow[t]{2}{*}{ Age (years) } & $25-54$ vs $18-24,1.12(1.01,1.25)$ & $25-54$ vs $18-24,1.22(1.10,1.34)$ \\
\hline & $55-64$ vs $18-24,1.21(1.04,1.40)$ & $55-64$ vs $18-24,1.17(1.02,1.33)$ \\
\hline \multirow[t]{2}{*}{ Sex } & Male vs female, $0.84(0.78,0.91)$ & Male vs female, $0.93(0.87,1,00)$ \\
\hline & Other vs female, $0.22(0.14,0.33)$ & Other vs female, $0.68(0.45,1.03)$ \\
\hline \multirow[t]{3}{*}{ Income } & $\$ 2-\$ 8$ vs $<\$ 2,1.38(1.09,1.74)$ & $\$ 2-8$ vs $<\$ 2,0.91(0.72,1.14)$ \\
\hline & $\$ 32+v s<\$ 2,2.18(1.79,2.64)$ & $\$ 32+$ vs $<\$ 2,1.47(1.21,1.79)$ \\
\hline & Refused vs $<\$ 2,0.91(0.71,1.16)$ & Refused vs $<\$ 2,0.78(0.61,1.00)$ \\
\hline \multirow[t]{3}{*}{ Education } & Medium vs low, $1.26(1.15,1.39)$ & Medium vs low, $1.26(1.15,1.37)$ \\
\hline & High vs low, $1.34(1.21,1.48)$ & High vs low, $1.24(1.13,1.36)$ \\
\hline & Very high vs low, $1.45(1.25,1.69)$ & Very high vs low, $1.31(1.15,1.49)$ \\
\hline \multirow[t]{2}{*}{ Mortality per million population } & Middle vs low, $1.38(1.25,1.52)$ & Middle vs low, $0.61(0.56,0.67)$ \\
\hline & High vs low, $1.43(1.30,1.56)$ & High vs low, $0.66(0.61,0.72)$ \\
\hline Trust in government & Yes vs no, $1.67(1.54,1.80)$ & Yes vs no, $4.35(4.01,4.72)$ \\
\hline
\end{tabular}

Arguably, trust is an intrinsic and potentially modifiable component of successful uptake of a COVID-19 vaccine. Our findings show that trust in government is strongly associated with vaccine acceptance and can contribute to public compliance with recommended actions ${ }^{15}$. Lessons learned from previous infectious disease outbreaks and public health emergencies, including HIV, H1N1, SARS, MERS and Ebola, remind us that trusted sources of information and guidance are fundamental to disease control ${ }^{16}$. However, addressing vaccine hesitancy requires more than building trust. It is a multifactorial, complex and context-dependent endeavor that must be addressed simultaneously at global, national and sub-national levels.

Clear and consistent communication by government officials is crucial to building public confidence in vaccine progams. This includes explaining how vaccines work, as well as how they are developed, from recruitment to regulatory approval based on safety and efficacy. Effective campaigns should also aim to carefully explain a vaccine's level of effectiveness, the time needed for protection (with multiple doses, if required) and the importance of population-wide coverage to achieve community immunity. Instilling public confidence in regulatory agency reviews of vaccine safety and effectiveness will be important. Credible and culturally informed health communication is vital in influencing positive health behaviors ${ }^{17,18}$, as has been observed with respect to encouraging people to cooperate with COVID-19 control measures. This includes preparing the public and leaders of civic, religious and fraternal organizations that are respected within various sectors of society and local communities, as well as the private sector, for a mass vaccination program with credible spokespeople, local engagement, accurate information and technological support.

This study had limitations. It must be kept in mind that all public surveys of the type reported here are snapshots taken at a point in time. This particular survey was conducted in the context of a highly dynamic and changing landscape, with daily variations in perceived disease threat and COVID-19 vaccine development itself.

In the 3 months since this survey was conducted, several highly visible events have affected COVID-19 vaccine development. A phase III trial of one of the most visible vaccine candidates was put on hold due to the report of a serious adverse event, although that hold was later lifted ${ }^{19}$. The Russian government, on the other hand, has made a vaccine available for general use on the basis of extremely limited data ${ }^{20}$. A group of former US Food and Drug Adminstration commissioners and several companies developing COVID-19 vaccines and biomedical advisory bodies have urged the US federal government to base any pending vaccine approvals on clinical data and not bend to political pressure from the incumbent US president to approve a vaccine before the US elections (https://www.washingtonpost.com/ opinions/2020/09/29/ former-fda-commissioners-coronavirus- vaccine-trump/).

In the context of these developments that post-date our survey, subsequent national surveys in the United States and elsewhere have suggested strongly that public hesitancy to a COVID-19 vaccine is now greater than that which we report here (https://www.kff.org/ coronavirus-covid-19/ press-release/poll-most- americans-worrypolitical-pressure- will-lead-to-premature-approval- of-a-covid-19vaccine-half- say-they-would-not-get- a-free-vaccine-approvedbefore-election-day/). This can only intensify the vaccine communications challenges discussed above.

In most of the 19 countries surveyed in our study, current levels of willingness to accept a COVID-19 vaccine are insufficient to meet the requirements for community immunity. To build increased trust among the general population, the elements that define and build trust must be understood and interventions crafted accordingly. It is increasingly clear that transparent, evidence-informed policy and clear, accurate communication will be required of all relevant stakeholders. The ongoing pandemic provides the public health community with an important opportunity to build vaccine literacy and confidence to support the uptake of a potential COVID-19 vaccine, as well as to bolster overall immunization programs for all vaccine-preventable diseases.

\section{Online content}

Any methods, additional references, Nature Research reporting summaries, source data, extended data, supplementary information, acknowledgements, peer review information; details of author contributions and competing interests; and statements of 
data and code availability are available at https://doi.org/10.1038/ s41591-020-1124-9.

Received: 24 August 2020; Accepted: 8 October 2020; Published online: 20 October 2020

\section{References}

1. MacDonald, N. E. \& SAGE Working Group on Vaccine Hesitancy. Vaccine hesitancy: definition, scope and determinants. Vaccine 33, 4161-4164 (2015).

2. Karafillakis, E., Larson, H. J. \& ADVANCE Consortium. The benefit of the doubt or doubts over benefits? A systematic literature review of perceived risks of vaccines in European populations. Vaccine 35, 4840-4850 (2017).

3. Cobos Muñoz, D., Monzón Llamas, L. \& Bosch-Capblanch, X. Exposing concerns about vaccination in low- and middle-income countries: a systematic review. Int. J. Public Health 60, 767-780 (2015).

4. European Parliament. European Parliament resolution of 19 April 2018 on vaccine hesitancy and drop in vaccination rates in Europe (2017/2951 RSP). https://www.europarl.europa.eu/ doceo/document/TA-8-2018-0188_EN.pdf (2018).

5. Larson, H. J., Jarrett, C., Eckersberger, E., Smith, D. M. D. \& Paterson, P. Understanding vaccine hesitancy around vaccines and vaccination from a global perspective: a systematic review of published literature, 2007-2012. Vaccine 32, 2150-2159 (2014).

6. Lane, S., MacDonald, N. E., Marti, M. \& Dumolard, L. Vaccine hesitancy around the globe: analysis of three years of WHO/UNICEF Joint Reporting Form data-2015-2017. Vaccine 36, 3861-3867 (2018).

7. Enserink, M. \& Cohen, J. Fact-checking Judy Mikovits, the controversial virologist attacking Anthony Fauci in a viral conspiracy video. Science https://www.sciencemag.org/ news/2020/05/fact-checking- judy-mikovitscontroversial- virologist-attacking-anthony- fauci-viral (2020).

8. Cornwall, W. Officials gird for a war on vaccine misinformation. Science 369, 14-19 (2020).
9. Fadda, M., Albanese, E. \& Suggs, L. S. When a COVID-19 vaccine is ready, will we all be ready for it? Int. J. Public Health 65, 711-712 (2020).

10. Larson, H. J. et al. Measuring trust in vaccination: a systematic review. Hum. Vaccines Immunother. 14, 1599-1609 (2018).

11. Biasio, L. R. Vaccine hesitancy and health literacy. Hum. Vaccines Immunother. 13, 701-702 (2017)

12. Thomson, A., Vallée-Tourangeau, G. \& Suggs, L. S. Strategies to increase vaccine acceptance and uptake: from behavioral insights to context-specific, culturally-appropriate, evidence-based communications and interventions. Vaccine 36, 6457-6458 (2018).

13. European Centre for Disease Prevention and Control. Catalogue of interventions addressing vaccine hesitancy. https://www.ecdc.europa.eu/ sites/ portal/files/documents/ Catalogue-interventions- vaccine-hesitancy.pdf (2017).

14. Lugo, N. R. Will carrots or sticks raise influenza immunization rates of health care personnel? Am. J. Infect. Control 35, 1-6 (2007).

15. Hovland, C. I. \& Weiss, W. The influence of source credibility on communication effectiveness. Public Opin. Q. 15, 635 (1951).

16. Siegrist, M. \& Zingg, A. The role of public trust during pandemics: implications for crisis communication. Eur. Psychol. 19, 23-32 (2014)

17. Quinn, S. C. et al. Exploring communication, trust in government, and vaccination intention later in the $2009 \mathrm{H} 1 \mathrm{~N} 1$ pandemic: results of a national survey. Biosecurity Bioterrorism 11, 96-106 (2013).

18. Shore, D. A. Communicating in times of uncertainty: the need for trust. J. Health Commun. 8, 13-14 (2003).

19. Mahase, E. Covid-19: Oxford researchers halt vaccine trial while adverse reaction is investigated. BMJ 370, m3525 (2020).

20. Burki, T. K. The Russian vaccine for COVID-19. Lancet Respir. Med. https://doi.org/10.1016/S2213-2600(20)30402-1 (2020).

Publisher's note Springer Nature remains neutral with regard to jurisdictional claims in published maps and institutional affiliations.

(C) The Author(s), under exclusive licence to Springer Nature America, Inc. 2020, corrected publication 2021 


\section{Methods}

We analyzed two questions from the COVID-SCORE study pertaining to COVID19 vaccine acceptance ${ }^{21}$. In that survey, participants responded to a total of 22 items, including two related to vaccine uptake, one related to trust in pandemic information sources and standard demographic questions regarding age, gender, level of education and household income (Supplementary Table 1).

Study participants. Participants were recruited by Consensus Strategies through multiple international online panel providers for each country to avoid coverage bias: Dynata provided 7,423 respondents across all 19 countries; Opinion Access provided 3,293 respondents from 14 countries; Survey Monkey provided 1,941 responses from 12 countries; and Amazon MTurk provided 762 respondents from eight countries. Respondents' identities were verified using IP addresses and mobile phone numbers to ensure that each participant was real and unique upon initial registration. Participants were recruited for the panels via a variety of methods, including online, telephone and direct mail solicitation. Sampling was random and is described in detail elsewhere ${ }^{21}$.

This study was approved by Emerson College, USA (institutional review board protocol no. 20-023-F-E-6/12), with an expiration date of June 11, 2021. The online questionnaire was administered by Emerson College to gather information from respondents after obtaining their written, informed consent about the survey and this project. No personally identifiable information was collected or stored.

Data collection. Survey data were collected from June 16 to June 20, 2020, from an online panel of 13,426 respondents aged 18 years or older from 19 countries from among the top 35 countries affected by the pandemic in terms of cases per million population, ranging between 619 and 773 participants per country. To ensure regional representation, we selected the next most affected country from regions not represented on the top 35 list: Brazil, Canada, China, Ecuador, France, Germany, India, Italy, Mexico, Nigeria, Poland, Russia, Singapore, South Africa, South Korea, Spain, Sweden, the United Kingdom and the United States ${ }^{22}$.

The more general vaccine-related question was, 'If a COVID-19 vaccine is proven safe and effective and is available to me, I will take it'. Respondents were also asked to register their level of agreement with a second statement: 'I would follow my employer's recommendation to get a COVID-19 vaccine once the government has approved it as safe and effective'. Responses were recorded on a five-point Likert scale ('completely disagree,' 'somewhat disagree', 'neutral/no opinion', 'somewhat agree' and 'completely agree'). We examined the demographic breakdown of the responses to these questions. Data for age and income were collected through open-text fields. Age was coded into age categories: 18-24, $25-54,55-64$ and 65 years or older. Where respondents provided income information, the levels were categorized as ' $<$ (US) $\$ 2$ per day', ' $\$ 2-\$ 8$ per day', ' $\$ 8-\$ 32$ per day' and ' $\$ 32+$ per day'. Education levels were categorized as less than high school (low), high school or some college (medium), bachelor's degree (high) and postgraduate degree (very high). Gender was defined as male, female or other. We also collected information on whether the respondent or a family member had been sick with COVID-19 and COVID-19 cases and deaths per million population at the country level ${ }^{22}$. For cases per million population and mortality per million population, we categorized the continuous values into categories of low, medium and high. For cases per million population, low was defined as fewer than 2,000 cases per million population; medium was defined as between 2,000 and 4,000 cases per million population; and high was defined as more than 4,000 cases per million population. For mortality per million population, low was defined as fewer than 200 deaths per million population; medium was defined as between 200 and 400 deaths per million population; and high was defined as more than 400 deaths per million population.
Analysis. We analyzed the distribution of the responses against the different questions for the entire dataset and further examined differences by country. We calculated results for two sets of univariate regressions: one for each of the two questions related to vaccines. We used logistic regression, defining the outcome as 1 if a respondent answered 'completely agree' or 'somewhat agree' and 0 for any other response. The independent demographic variables included age, gender, income and education. We also examined the relationship between the two regression outcomes and whether someone in the respondent's family had been sick with COVID-19, as well as existing country-by-country data on COVID-19 cases per million population, COVID-19 mortality per million population and whether a respondent reported that they trusted pandemic information from their government (yes or no).

Reporting Summary. Further information on research design is available in the Nature Research Reporting Summary linked to this article.

\section{Data availability}

All data used in this analysis can be found at https://osf.io/kzq69/.

\section{Code availability}

All code to reproduce this analysis can be found at https://osf.io/kzq69/.

\section{References}

21. Lazarus, J. V. et al. COVID-SCORE: a global survey to assess public perceptions of government responses to COVID-19 (COVID-SCORE-10). PLoS ONE 15, e0240011 (2020).

22. Johns Hopkins Coronavirus Resource Center. COVID-19 dashboard. https:// coronavirus.jhu.edu/map.html (2020).

\section{Acknowledgements}

Funding was provided by the City University of New York Graduate School of Public Health and Health Policy Foundation, Bocconi University, J. Fielding, the United States Council for International Business Foundation and K. Rabin. Consensus Strategies was the funding recipient. J.V.L. acknowledges support to ISGlobal from the Spanish Ministry of Science, Innovation and Universities through the 'Centro de Excelencia Severo Ochoa 2019-2023' Programme (CEX2018-000806-S) and from the Government of Catalonia through the CERCA Programme.

\section{Author contributions}

S.C.R., J.V.L. and A.E.M. conceived the study. S.K. collected the data. A.P. was responsible for the coding of the analyses. A.P., A.E.M. and J.V.L. analyzed the data. J.V.L., A.P., K.R. and A.E.M. wrote the first draft of the paper. J.V.L., A.E.M., A.P., K.R., S.C.R., L.O.G. and H.L. edited and approved the final manuscript.

\section{Competing interests}

The authors declare no competing interests.

\section{Additional information}

Extended data is available for this paper at https://doi.org/10.1038/s41591-020-1124-9.

Supplementary information is available for this paper at https://doi.org/10.1038/ s41591-020-1124-9.

Correspondence and requests for materials should be addressed to J.V.L.

Reprints and permissions information is available at www.nature.com/reprints. 


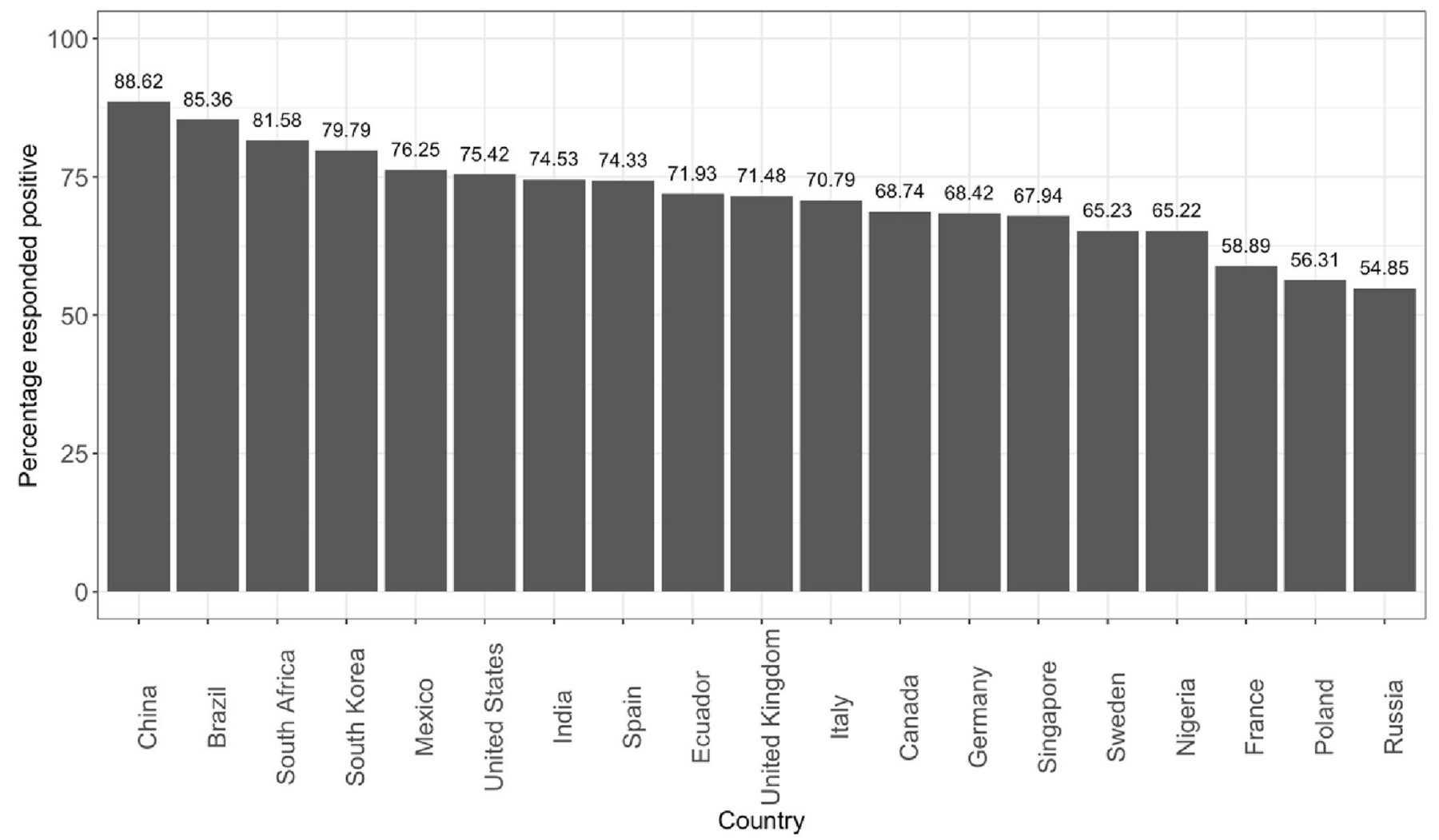

Extended Data Fig. 1 | 'If a COVID-19 vaccine is proven safe and effective and is available, I will take it'. Numbers above the bars represent the percent of respondents in each country who responded positively to the question 'If a COVID-19 vaccine is proven safe and effective and is available, I will take it'. 


\section{Reporting Summary}

Nature Research wishes to improve the reproducibility of the work that we publish. This form provides structure for consistency and transparency in reporting. For further information on Nature Research policies, see our Editorial Policies and the Editorial Policy Checklist.

\section{Statistics}

For all statistical analyses, confirm that the following items are present in the figure legend, table legend, main text, or Methods section.

$\mathrm{n} / \mathrm{a}$ Confirmed

$\bigotimes$ The exact sample size $(n)$ for each experimental group/condition, given as a discrete number and unit of measurement

$\bigotimes$ A statement on whether measurements were taken from distinct samples or whether the same sample was measured repeatedly

The statistical test(s) used AND whether they are one- or two-sided

Only common tests should be described solely by name; describe more complex techniques in the Methods section.

$\bigotimes$ A description of all covariates tested

Х A description of any assumptions or corrections, such as tests of normality and adjustment for multiple comparisons

$\triangle$ A full description of the statistical parameters including central tendency (e.g. means) or other basic estimates (e.g. regression coefficient)

AND variation (e.g. standard deviation) or associated estimates of uncertainty (e.g. confidence intervals)

Х $\square \begin{aligned} & \text { For null hypothesis testing, the test statistic (e.g. } F, t, r \text { ) with confidence intervals, effect sizes, degrees of freedom and } P \text { value noted } \\ & \text { Give } P \text { values as exact values whenever suitable. }\end{aligned}$

Х $\square$ For Bayesian analysis, information on the choice of priors and Markov chain Monte Carlo settings

$\bigotimes \square$ For hierarchical and complex designs, identification of the appropriate level for tests and full reporting of outcomes

$\triangle \square$ Estimates of effect sizes (e.g. Cohen's d, Pearson's $r$ ), indicating how they were calculated

Our web collection on statistics for biologists contains articles on many of the points above.

\section{Software and code}

Policy information about availability of computer code

Data collection No software was used for data collection.

Data analysis Authors used R.

For manuscripts utilizing custom algorithms or software that are central to the research but not yet described in published literature, software must be made available to editors and

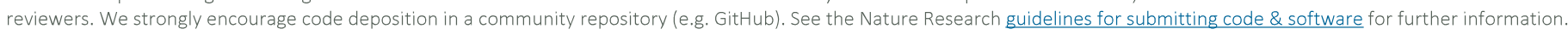

\section{Data}

Policy information about availability of data

All manuscripts must include a data availability statement. This statement should provide the following information, where applicable:

- Accession codes, unique identifiers, or web links for publicly available datasets

- A list of figures that have associated raw data

- A description of any restrictions on data availability

All the data used in this analysis can be found at https://osf.io/kzq69/. 
Please select the one below that is the best fit for your research. If you are not sure, read the appropriate sections before making your selection.

Life sciences

Behavioural \& social sciences

Ecological, evolutionary \& environmental sciences

For a reference copy of the document with all sections, see nature.com/documents/nr-reporting-summary-flat.pdf

\section{Behavioural \& social sciences study design}

All studies must disclose on these points even when the disclosure is negative.

Study description

Cross-sectional random sample general population survey of 13,426 individuals in 19 countries using quantitative methods.

Research sample

A representative sample of the general adult population in Brazil, Canada, China, Ecuador, France, Germany, India, Italy, Mexico, Nigeria, Poland, Russia, Singapore, South Africa, South Korea, Spain, Sweden, the United Kingdom, and the United States according to each countries' age, gender, educational level, income level and regional distribution, as outlined in the sampling strategy below.

Sampling strategy

Strata were established by age (using the following age groups: 18-24, 25-54, 55-64 and 65 years and older); gender (male, female, transgender, and "other,"); and level of education (based on each country's educational system), which was calculated from data provided by UNESCO, the Organisation for Economic Co-operation and Development, and country data from Sweden, the United Kingdom, and the United States. Educational level was coded into three groups of low, medium and high. "Low" included people who reported not finishing a secondary education (high school); "medium" included those who had completed secondary, vocational, technical, professional associate or high school degree; the "high" group consisted of those who had completed a tertiary or bachelor's degree and postgraduate work. Each country was divided into regions based on city/town, province or state unit of analysis. The number of participants who could enrol in each of these strata was calculated to reflect the distribution in the general population based on census/survey estimates provided by the World Bank and CIA World Factbook. Data were weighted by strata with each stratum requiring a minimum of 50 participants.

Data collection $\quad$ Participants were recruited through multiple international online panel providers for each country to avoid coverage bias. Respondents' identities were verified using IP addresses and their mobile phones were verified to ensure that each participant was real and unique upon initial registration. Participants were recruited for the panels via a variety of methods, including online, telephone and direct mail solicitation, and equtiably compensated regardless of country being polled in order to comply with ethical compensation standards. All data were collected via online, telpehone or mail-based survey. Participants were recruited for the panels via online, telephone or direct mail solicitation. Researchers were blinded to the experimental conditation and study hypothesis.

Timing

Survey data were collected from 16 to 20 June 2020

Data exclusions

Based on sampling strategy if a strata was full or individual did not meet the age requirement

Non-participation

No participants declined participation.

Randomization

Strata were established by age (using the following age groups: 18-24, 25-54, 55-64 and 65 years and older); gender (male, female, transgender, and "other,"); and level of education (based on each country's educational system), which was calculated from data provided by UNESCO, the Organisation for Economic Co-operation and Development, and country data from Sweden, the United Kingdom, and the United States. Educational level was coded into three groups of low, medium and high. "Low" included people who reported not finishing a secondary education (high school); "medium" included those who had completed secondary, vocational, technical, professional associate or high school degree; the "high" group consisted of those who had completed a tertiary or bachelor's degree and postgraduate work. Each country was divided into regions based on city/town, province or state unit of analysis. The number of participants who could enrol in each of these strata was calculated to reflect the distribution in the general population based on census/survey estimates provided by the World Bank and CIA World Factbook. Data were weighted by strata with each stratum requiring a minimum of 50 participants.

\section{Reporting for specific materials, systems and methods}

We require information from authors about some types of materials, experimental systems and methods used in many studies. Here, indicate whether each material, system or method listed is relevant to your study. If you are not sure if a list item applies to your research, read the appropriate section before selecting a response. 
Materials \& experimental systems

Methods

$\mathrm{n} / \mathrm{a}$ Involved in the study $\mathrm{n} / \mathrm{a}$ Involved in the study

\ $\square$ Antibodies

Х $\square$ ChIP-seq

\ $\square$ Eukaryotic cell lines

Х $\square$ Flow cytometry

Х $\square$ Palaeontology and archaeology

Х $\square$ MRI-based neuroimaging

$\bigotimes \square$ Animals and other organisms

$\square$ \uman research participants

\ $\square$ Clinical data

$\bigotimes \square$ Dual use research of concern

\section{Human research participants}

Policy information about studies involving human research participants

$\begin{array}{ll}\text { Population characteristics } & \text { See above } \\ \text { Recruitment } & \text { See above } \\ \text { Ethics oversight } & \text { This study was approved by Emerson College, USA (IRB protocol number 20-023-F-E6/12). }\end{array}$

Note that full information on the approval of the study protocol must also be provided in the manuscript. 